\title{
Steady-State Responses and Stability Analyses of the Duffing's Oscillator with the Softening Spring to an External Exciting Force*
}

\author{
Yoshihiro TSUDA**, Miin-nan HUANG*** \\ and Atsuo SUEOKA****
}

\begin{abstract}
The steady-state responses which the damped Duffing oscillator with a softening spring property under a harmonically stimulating force exhibited in the main resonant frequency region have been analyzed by using both an approximate analytical procedure; that is, the harmonic balance scheme, and a numerical procedure. With regard to the structure which the system reveals in this region, there exists another branch which seems to be caused by bifurcation from the resonant branch at a certain frequency as well as to the usual resonant and nonresonant branches. It has been verified that many kinds of periodic solutions exist in the frequency region of interest between this new branch and the resonant one. In addition to the traditional harmonic solutions; that is, resonant and nonresonant solutions and the new branch, there appears a third harmonic solution branch. However, it is absolutely unstable. This unstable solution enables us to explain reasonably the constitution of attractors in this resonant frequency region. Furthermore, an approximate stability analysis of these new branches, including the usual resonant and nonresonant branches, has been developed and has revealed stability problems of all these branches. It has also been shown that chaotic phenomena, which are related to the fundamental harmonics in the region of interest, arise due to period doubling bifurcation and develop only along the path of the second new branch.
\end{abstract}

Key Words: Nonlinear Vibration, Forced Vibration, Duffing System, Numerical Analysis, Approximate Stability Analysis, Harmonic Balance Method, Chaos, Basin of Attraction

\section{Introduction}

The Duffing's oscillator with a damper is one of the systems that has attracted a great deal of attention and has been studied extensively, since the system is fundamental and is also important in analyzing and in doing research on nonlinear vibrating phenomena. In the system with hardening type $\mathrm{e}^{(1),(2)}$ and softening type of its elastic property, the behaviors in the region

* Received 9th November, 1997

** Dept. of Mechanical Engineering, Faculty of Engineering, Ohita University, 700 Dannoharu, Ohita 8701124, Japan

*** Graduate Student of Ohita University

**** Dept. of Mechanical Engineering, Faculty of Engineering, Kyushu University, 6-10-1 Hakozaki, Higasiku, Fukuoka 812-0053, Japan of the principal resonance, including chaotic motions, have been discussed by many researchers ${ }^{(3)-(8)}$, and have been clearly explained. For example, Dowell and Pezeshki ${ }^{(1)}$ presented the criterion of occurrence of chaotic behavior, and Pezeshki and Dowell ${ }^{(9)}$ showed the basin of attraction to chaotic responses by using the numerical integration procedure.

Schmidt-Tondl ${ }^{(7)}$ computed the domains of attraction of the stable harmonic solutions by making use of an analog computer which showed some significant results. They did not yield, however, a reasonable and clear explanation for the attraction, that is to say, separatrices of their trajectories.

Novak and Frehlich ${ }^{(2)}$ mentioned the scenario of occurrence of period-doubling bifurcation via symmetry breaking through an approximate analysis and an analog computer, but they did not sufficiently explain 
the causes of the phenomenon with regard to the characteristics of this system. Concerning the studies of these circumstances, Ref. ( 8 ) may be quoted. The reference ( 8 ) stated bifurcations of symmetric solutions into unsymmetric solutions and individual branch corresponding to these solutions, and explained that it may be possible that period doubling bifurcations along the branch corresponding to the unsymmetric solution result in the occurrence of many kinds of periodic solutions and aperiodic solutions. Without the application of an approximate procedure, all of the results ${ }^{(8)}$ are obtained by means of a computer simulation with a direct numerical integration. So it seems fairly difficult to foresee the characteristics of the system, for example, predictions of the influences of the individual system parameter on the responses. Szemplinska ${ }^{(10)}$ made an approximation of evolutions of symmetrical solutions into unsymmetrical solutions and also period doubling bifurcations, in the same manner as Refs. (2) and ( 8 ).

References (4) and (5) maintained that they may obtain high-precision results of nonlinear oscillators, i.e., the Duffing system, by using the Galerkin method. In particular, Leung ${ }^{(4)}$ reported the "perfect" response curves of the Duffing system.

Although the vibrating system under consideration makes both symmetrical and unsymmetrical solutions, all of the studies cited above bear only the symmetrical solutions in mind. Investigations without both these solutions merely result in inaccurate conclusions or erroneous understanding of the behavior of the system. The lack of understanding of the unsymmetrical solutions has caused an erroneous route to chaos in the main resonant region ${ }^{(10)-(14)}$. Besides, stability analyses of approximate, analytical solutions may be imperfect in this region due to the fact that the results of stability analyses do not coincide with actual responses, leaving the subject still open to discussion.

Therefore, making clear the characteristic of a damped Duffing oscillator is extremely important in the field of nonlinear dynamics as well as the nonlinear theory. This also seems to be very important matter from a viewpoint of engineering.

In the same manner of Refs. (1),(2) and (6) to (10), this paper has dealt with the damped Duffing system, and, in particular, investigated both details of the characteristic of this system, including the precise approximate stability analysis, and chaotic phenomena at the region of the principal resonance by means of approximate analytical procedure, i.e., the harmonic balance method, and numerical simulations, bearing the results of references ${ }^{(4)-(6),(11)}$ in mind.

As a result, it has been clearly shown that there exist many kinds of periodic solutions, i.e., harmonic and subharmonic solutions and chaotic motions in the frequency region under consideration, and the characteristic may be much more complex than previous research. Furthermore, an approximate stability analysis developed has brought about the stabilities of the fundamental harmonics qualitatively and quantita. tively.

\section{Approximate Analyses}

\section{1 Steady-state periodic solutions}

Let's consider the following one-degree-of-freedom vibrating system, i.e., the softening Duffing's oscillator with a damper, which has been analyzed previously in many fields ${ }^{(15)}$.

$$
x^{\prime \prime}+h x^{\prime}+k x+q x^{3}=a \cos \omega t
$$

where $h$ is coefficient of viscous damping, $k$ coefficient of linear spring, $q$ coefficient of nonlinear spring, $a$ amplitude of exciting external force, $\omega$ frequency of the force, $(\quad)^{\prime}=d(\quad) / d t$ and $T=2 \pi / \omega$.

The approximate solution of harmonic motions of Eq. (1) may be assumed in the form ${ }^{(3),(16)}$;

$$
x=u \cos \omega t+v \sin \omega t
$$

where $u$ and $v$ are amplitudes of harmonics. Reference (11) presents, as a more accurate solution than the expression given by Eq. (2), an approximate solution as follows:

$$
x=R+u \cos \omega t+v \sin \omega t
$$

where $R$ is a constant component of the response. However, the expression of Ref. (11) is erroneous, and Eqs. (2) and (3) are different from each other, though they lead to the same fundamental solution. That is, Eq. ( 2 ) corresponds to a symmetric solution and Eq. ( 3 ) to an unsymmetrical one; and, however hard one may try, Eq. ( 3 ) could not generate a high accurate solution which should improve the precision of Eq. (2), as far as $R \neq 0$. Reference(11) did not carry out a practical computation for Eq. (3), including an examination of its stability. For the sake of convenience, Eq. (2) is referred to as the second approximate solution and Eq. (3) as the third one, according to the number of unknown parameters involved. The characteristic and stability of Eqs. (2) and ( 3 ) will be explained in detail below. As Eq. ( 3 ) with the case of $R=0$ naturally is equivalent to Eq. (2), the quality of the fundamental harmonics is investigated, using Eq. ( 3 ).

Utilizing the harmonic balance $\operatorname{method}^{(17)}$ provides the following equations ;

$$
\begin{aligned}
& R\left\{k+q\left[R^{2}+(3 / 2)\left(u^{2}+v^{2}\right)\right]\right\}=0, \\
& \left(k-\omega^{2}\right) v-\omega h u+3 q v\left[R^{2}+\left(u^{2}+v^{2}\right) / 4\right]=0, \\
& \left(k-\omega^{2}\right) u+\omega h v+3 q u\left[R^{2}+\left(u^{2}+v^{2}\right) / 4\right]-a=0
\end{aligned}
$$

From Eq. (4), the steady-state solution is obtained; 


$$
\begin{aligned}
& R\left\{k+q\left(R^{2}+3 r^{2} / 2\right)\right\}=0, \\
& \left(h^{2} \omega^{2}+\Phi^{2}\right) r^{2}=a^{2}
\end{aligned}
$$

where $r^{2}=u^{2}+v^{2}, \quad \Phi=k-\omega^{2}+3 q R^{2}+3 q r^{2} / 4$

In consideration of the first equation of Eq. (5), we can easily see that there may exist two kinds of steady-state harmonic solutions which correspond to $R=0$ and $R \neq 0$, respectively. Taking Eq. ( 3 ) into consideration, the steady-state solution corresponding to $R=0$ may be defined as symmetric, while the one corresponding to $R \neq 0$ as unsymmetrical. In order to clarify the characteristic of the nonlinear vibrating system given by Eq. ( 1 ) in the main resonant region, it is necessary to investigate not only the existence and behavior but also the stability of these two steadystate solutions.

\subsection{Stability analyses}

$x_{0}(t)$ is defined as the steady-state solution (see Eq. (3)) in the previous section. Although there have been many previous papers ${ }^{(10),(13),(18)-(20)}$ which have analyzed the stability of the steady-state solution $x_{0}(t)$, their results ${ }^{(10) \sim(12)}$ have seemed insufficient. Defining $\xi(t)$ as the small disturbance to $x_{0}(t)$, the (approximate) variational equation for Eq. (1) becomes as follows:

$$
\xi^{\prime \prime}+h \xi^{\prime}+\left(k+3 q x_{0}^{2}\right) \xi=0
$$

\subsubsection{Stability-numerical integration method} Equation( 6 ) is the well-known Hill's type equation $^{(21)}$. No general procedure which provides its characteristic exponents is known ${ }^{(22)}$. As one of the practical methods of computing these exponents to determine the stability of the solution $x_{0}(t)^{(23)}$, the following procedure is used: to numerically integrate a fundamental matrix solution over one period, $\mathrm{T}$, with unit matrix as its initial value ${ }^{(23) \sim(25)}$. For the sake of convenience, this procedure is defined as the "numerical integration method". As shown in Chap. 3 below, the reliability of this procedure seems to be somewhat poor ${ }^{(26)}$, while it is quite convenient.

2.2.2 Stability-Hayashi method The stability analysis by Ref.(19) is defined as "Hayashi method", for simplicity. Thus transformation of variables from $\xi(t)$ to $\eta(t)$ in Eq. ( 6$)$ leads to:

$$
\eta^{\prime \prime}+F(t) \eta=0
$$

where $F(t)$ is a periodic function with period, $T$ and $\eta(t)$ is also a periodic function with period, $T^{(23),(27),(28)}$.

a. Symmetric Solution: Case of $R=0$

The response curves for the symmetric case may be obtained from the second equation of Eq. (5), where $R=0$. The variational equation, Eq. (7), of this case is as follows:

$$
\eta^{\prime \prime}+\left[\theta_{0}+2 \theta_{1} \cos (2 \omega t-\varepsilon)\right] \eta=0
$$

where

$$
\begin{aligned}
& \xi=\eta \cdot \exp (-h t / 2), \quad \theta_{0}=k-h^{2} / 4+3 q r_{0}^{2} / 2, \\
& \theta_{1}=3 q r_{0}^{2} / 4,
\end{aligned}
$$

and $\varepsilon$ is a phase angle.

By applying the Floquet theory ${ }^{(10),(19)}$, one can easily find the first and second order unstable regions $^{(2),(19)}$.

The result is as follows : the first stability limit is given by the point of vertical tangent and the second stability limit is expressed by the condition ;

$$
\begin{aligned}
f_{1} & =\left(\theta_{0}+\mu^{2}\right)\left(\theta_{0}+\mu^{2}-4 \omega^{2}\right)^{2}-2 \theta_{1}^{2}\left(\theta_{0}+\mu^{2}-4 \omega^{2}\right) \\
& +\left.16 \mu^{2} \omega^{2}\left(\theta_{0}+\mu^{2}\right)\right|_{\mu=h / 2}=0
\end{aligned}
$$

b. Unsymmetric Solution: Case of $R \neq 0$

The response curves for this case are given by Eq. (5). The stability analysis of this case may be carried out in the same manner as the previous symmetric case, although there are a few differences from the previous case. The gist of this analysis is as follows :

The linear variational equation, Eq. ( 7 ), of this case becomes

$$
\eta^{\prime \prime}+\left[\theta_{0}+2 \sum_{n=1}^{2} \theta_{n} \cos \left(n \omega t-\varepsilon_{n}\right)\right] \eta=0
$$

where

$$
\begin{aligned}
& \theta_{0}=k-h^{2} / 4+3 q\left(R_{0}^{2}+r_{0}^{2} / 2\right), \\
& \theta_{1}=3 q R_{0} r_{0}, \quad \theta_{2}=3 q r_{0}^{2} / 4,
\end{aligned}
$$

and $\varepsilon_{n}^{\prime}$ s are the phase angles.

In order to determine the first unstable region the following is assumed ${ }^{(10),(19)}$;

$$
\eta=\exp (\mu t) \cdot \sin (\omega t / 2-\sigma)
$$

then, from Eqs. (10) and (11), the first stability limit is expressed as

$$
\begin{aligned}
f_{2} & =\left\{\theta_{0}-(\omega / 2)^{2}\right\}^{2}+2\left\{\theta_{0}+(\omega / 2)^{2}\right\}\left(h^{2} / 4\right) \\
& +h^{4} / 16-\theta_{1}^{2}=0
\end{aligned}
$$

The second unstable region may be calculated in the form ;

$$
\eta=\exp (\mu t)\{b+\sin (\omega t-\sigma)\}
$$

then the second stability limit becomes

$$
\wedge(h / 2)=0
$$

where

$$
\wedge(\mu)=\left|\begin{array}{ccc}
\theta_{0}+\mu^{2} & \theta_{1 s} & \theta_{1 c} \\
2 \theta_{1 s} & \theta_{0}+\mu^{2}-\theta_{2 c}-\omega^{2} & -2 \mu \omega+\theta_{2 s} \\
2 \theta_{1 c} & 2 \mu \omega+\theta_{2 s} & \theta_{0}+\mu^{2}+\theta_{2 c}-\omega^{2}
\end{array}\right|
$$

and furthermore

$$
\begin{aligned}
& \theta_{1 c}=3 q R u, \quad \theta_{1 s}=3 q R v, \\
& \theta_{2 c}=3 q\left(u^{2}-v^{2}\right) / 4, \quad \theta_{2 s}=3 q u v / 2
\end{aligned}
$$

Expanding and arranging Eq. (14), the second stability limit could be found to correspond to the point of vertical tangents of the response curves: that is

$$
\Phi^{2}-15 q r_{0}^{2} \Phi / 2+h^{2} \omega^{2}=0
$$

where $\Phi$ is referred to Eq. ( 5 ).

2.2.3 Stability-expansion method From Eq.

( 6 ), the following equation is assumed ${ }^{(23),(27),(28)}$ :

$$
\xi=\exp (\beta t) \cdot \eta(t)
$$

where $\beta=\mu-h / 2$ and $\mu$ is a characteristic exponent.

Through the process of expanding the periodic function $\eta(t)$ with period $T$ into the truncated Fourier 
series :

$$
\eta(t)=b_{0}+\sum_{n=1}^{L}\left(b_{n} \cos \left(n \omega t-\psi_{n}\right)\right)
$$

where $\phi_{n}^{\prime}$ s are the phase angles, and applying the harmonic balance method, the stability of $x_{0}(t)$ is determined $^{(26),(29),(30)}$.

For the sake of convenience, this scheme is defined as the "expansion method" and also, when Eq. (17) uses $n=L$, this analysis is defined as $(2 L+1)$ approximate stability analysis. As the result of computations, the determinant: $\Delta(\beta)^{(29)}$ with regard to the characteristic exponent $\beta$ was obtained as follows :

$$
\left.\Delta(\beta)\right|_{\beta=0}
$$

$$
\begin{gathered}
\left|\begin{array}{ccc}
\theta_{0} & 3 q \theta_{1 c} / 2 & 3 q \theta_{1 s} / 2 \\
3 q \theta_{1 c} & \theta_{0}-\omega^{2}+3 q \theta_{2 c} / 2 & \omega h+3 q \theta_{2 s} / 2 \\
3 q \theta_{1 s} & -\omega h+3 q \theta_{2 s} / 2 & \theta_{0}-\omega^{2}-3 q \theta_{2 c} / 2 \\
3 q \theta_{2 c} & 3 q \theta_{1 c} / 2 & -3 q \theta_{1 s} / 2 \\
3 q \theta_{2 s} & 3 q \theta_{1 s} / 2 & 3 q \theta_{1 c} / 2 \\
3 q \theta_{2 c} / 2 & 3 q \theta_{2 s} / 2 \\
3 q \theta_{1 c} / 2 & 3 q \theta_{1 s} / 2 \\
-3 q \theta_{1 s} / 2 & 3 q \theta_{1 c} / 2 \\
\theta_{0}-4 \omega^{2} & 2 \omega h \\
-2 \omega h & \theta_{0}-4 \omega^{2}
\end{array}\right|
\end{gathered}
$$

where

$$
\begin{aligned}
& \theta_{0}=k+3 q\left[R^{2}+\left(u^{2}+v^{2}\right) / 2\right], \\
& \theta_{1 c}=2 R u, \quad \theta_{1 s}=2 R v, \\
& \theta_{2 c}=\left(u^{2}-v^{2}\right) / 2, \quad \theta_{2 s}=u v
\end{aligned}
$$

Equation(18) corresponds to the case of $L=2$ and therefore the analysis to 5 th order approximation. The stability criterion is the following: $\Delta(\beta=0)>,=$, $<0$ corresponds to the stable, stability limit, unstable region, respectively ${ }^{(19),(29),(31)}$.

\section{Numerical Examples and Discussions}

The characteristic of the oscillator given by Eq. (1) in the main resonant frequency region has been investigated by both the approximate, analytical scheme as above-mentioned and the numerical simulation method of the previous work ${ }^{(32)}$ in order to obtain more precise behavior in comparison with the results provided by Eqs. (2) and (3). Solutions found through this numerical simulation will be referred to as "numerical solutions", while the solutions obtained through Eq. ( 3 ) will be referred to as "approximate solutions". Taking into account the results of Refs. (6) and (11), the values of system parameters used in this paper have been determined.

\section{1 Stability of fundamental harmonic solutions}

Figure 1 shows the steady-state response curves, obtained by the numerical simulation ${ }^{(32)}$, where solid lines and broken lines correspond to stable and unstable states, respectively. From the figure, the branches with the mark, (1), correspond to the symmetric solutions (i.e., resonant and nonresonant) and the branches with the mark, (2) and (3), to the unsymmetrical ones. As the frequency $\omega$ decreases $(\mathrm{H} \rightarrow \mathrm{A})$, the symmetric solution, branch (1), changes from the stable state into the unstable at point $\mathrm{A}$ (, corresponding to the interval $\mathrm{AC}$ ). At the same time, the unsymmetrical solution-branch (2) appeared from the point $A$ due to bifurcation (corresponding to symmetry breaking). This branch (2) contains the stable region, whose band width is quite narrow and is in the neighborhood of point $A$. The branch becomes unstable at the point $\mathrm{B}$ as the frequency $\omega$ decreases further.

As mentioned below (see section 3.2), the point A corresponds to the symmetry breaking and the point $B$ to period doubling bifurcation. Note that as the branch (1) with the unstable region $\mathrm{AC}$ is symmetric, the system can not exhibit phenomena of period doubling bifurcation, not to mention the chaotic phenomena along the path of this unstable region. The nature accompanied with the nonresonant branch (1) is quite simple, because the branch possesses only one turning point, that is, a point of saddle-node bifurcation.

Finally, although the branch (3) does not have a stable state in the whole frequency region and seems unnecessary in practice, it is needed to clarify reasonably the attractors of the responses of the system in the frequency region of interest on the phase plane, it is certainly necessary to take into consideration this unstable, apparently useless, branch (3). It should be noted that, since the branches (2) and (3) are unsymmetric solutions, there exist of course two solutions (positive and negative solution), i.e., two singular points on the phase plane, as explained in detail in section 3.3 below.

Comparing the response curves as shown in Fig. 1,

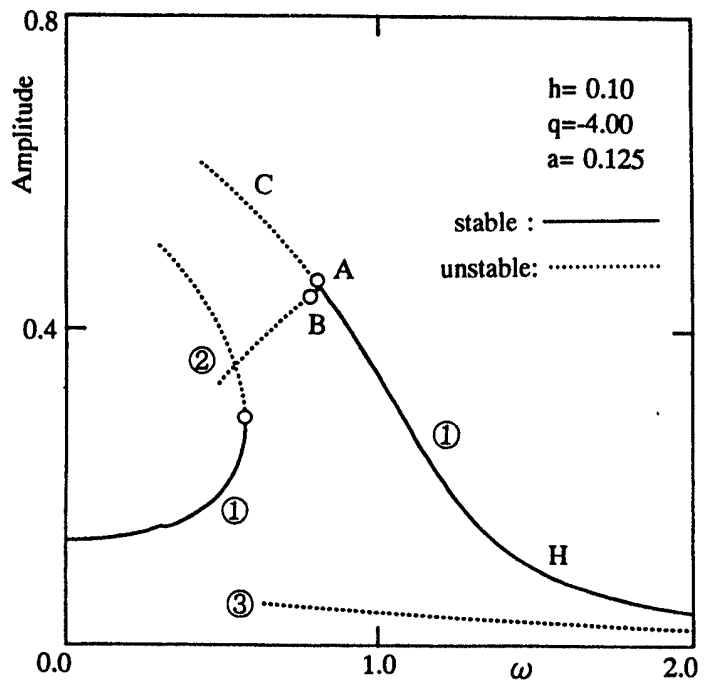

Fig. 1 Frequency response curve using "numerical procedure" 
let us consider the results, i.e., "approximate solutions", obtained through the approximate analyses.

In Fig. 2, shows the results obtained through the stability analysis-numerical integration method. Although the whole region of the section $\mathrm{AC}$ on the resonant, symmetrical branch must be unstable, this method has concluded that there exists some stable state near the point $\mathrm{C}$ as shown in Fig. 2. Also, the unsymmetric branch (2) should have a stable region near point A. Nevertheless, this method has indicated that the whole branch (2) is unstable. It is difficult for this method to be sufficiently effective and reliable with regard to stability analyses as in the same case with a hardening spring ${ }^{(29)}$, so applying this method to stability problems needs great care.

Figure 3 shows the results obtained by the stability analysis - Hayashi method. The curve, $\left(f_{1}\right)$, in Fig. 3(a) corresponds to Eq. (9), which is the second stability limit for the symmetric solution, and the curve, $\left(f_{2}\right)$, in Fig. 3( b ) to Eq. (12), which is the first stability limit for the unsymmetric one. Although stability criterion $\left(f_{2}\right)$ as to the unsymmetrical branch may seem to be valid, the criterion $\left(f_{1}\right)$ as to the symmetric one has determined that a region (section $\mathrm{CZ}$ in Fig. 3(a )) on the unstable, resonant symmetric branch near $\omega=0$ is stable, which is erroneous. See Fig. 1. References (10) and (12) have investigated the stability of the same system based on this method. However, their conclusions may be insufficient. Thus, it may be difficult to consider "Hayashi method" to be sufficiently reliable to the stability analysis.

The results obtained by the stability analysis expansion method are shown in Fig. 4. Figure 4(a), which shows apparently erroneous stabilities, corresponds to the results obtained by 3rd order analysis,

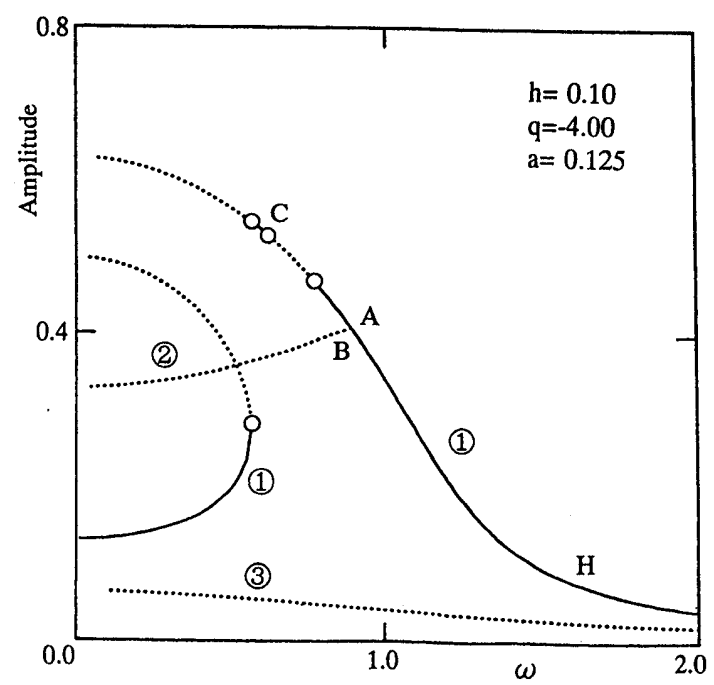

Fig. 2 Frequency response curve using "integration procedure" i.e., $L=1$ in Eq. (17). On the other hand, Fig. 4(b), which shows the result obtained by 5 th order analysis (i.e., $L=2$ ), agrees quite well with the results in Fig. 1 , qualitatively. The present order of approximation of the solution is 3rd order (see Eq. (3)) and the approximate stability analysis with the same order, i.e., 3rd order, can not bring about the reasonable and correct stability. In fact, it is necessary to use a higher order than 5 th order, in order to obtain the correct stability analysis. On the other hand, the precision of the stability has hardly been improved, even though the stability analysis adopts the higher order than 5th. Taking into consideration this situation, 5th approximate expression merely has been presented as to the order of approximation for the

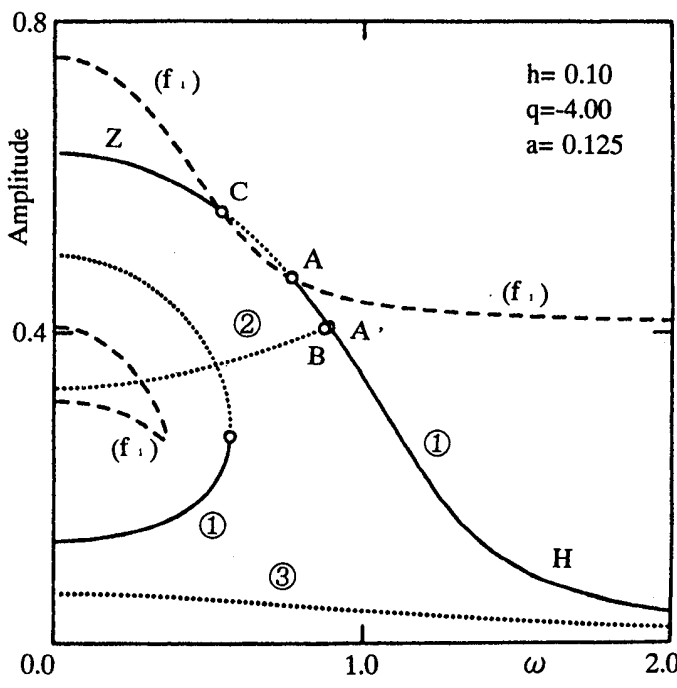

(a) Instability of the harmonic solution with the boundary $\left(f_{1}\right)$

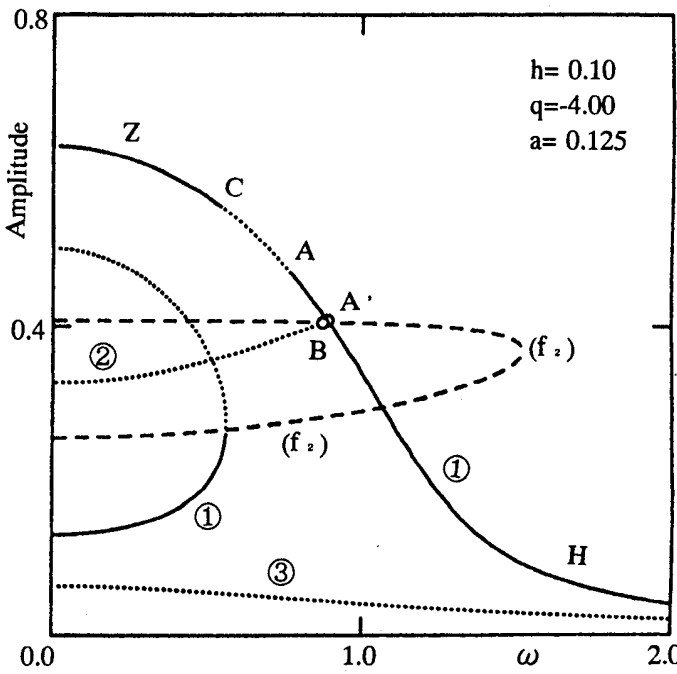

(b) Instability of the harmonic solution with the boundary $\left(f_{2}\right)$

Fig. 3 Frequency response curves using "approximate analytical procedure" 
determinant Eq.(18). Following the reference ${ }^{(26)}$, utilizing the order of approximation on stability analysis agreed with the order of approximate solution will bring about the correct result in the case of the system with hardening spring, while this criterion can not be equally applied for the system with softening spring and it is necessary to use the higher order of stability analysis than the order of the approximate solution.

Then, it is necessary to note that there exists the relation between the order of approximation of approximate solutions (e.g., Eqs.(2) and (3)) and that of approximate stability analyses (i.e., the value of $L$ ).

Judging from this viewpoint, the stability analyses of the system with the softening spring may be more difficult in comparison with the analyses of the

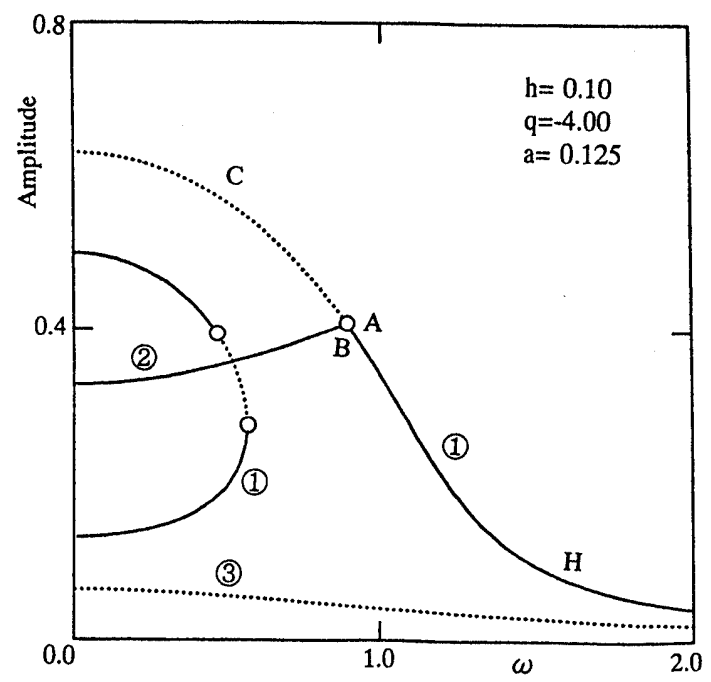

(a) Stability analysis with 3rd order

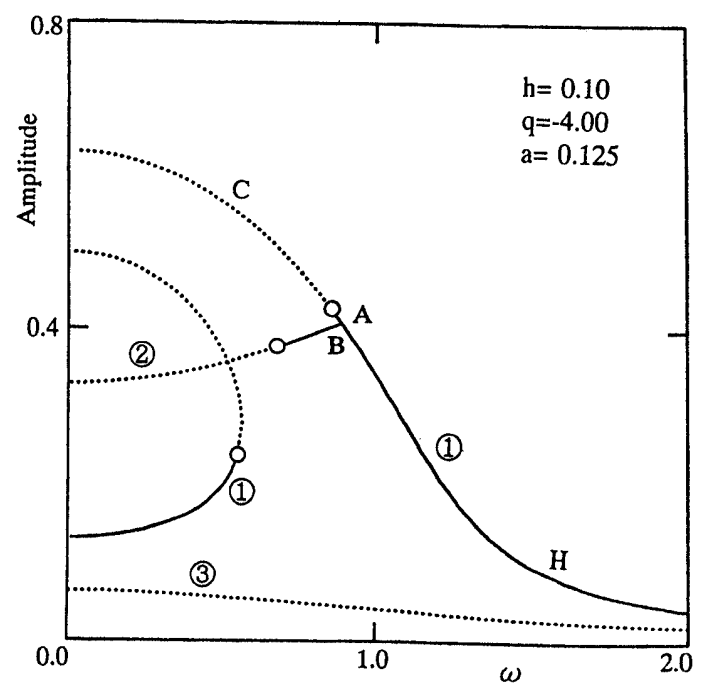

(b) Stability analysis with 5th order

Fig. 4 Frequency response curves using "expansion procedure" system with hardening spring.

The Hayashi method is essentially the same as the expansion method, although only the approximate precision of both methods is a little different from each other. However, this difference of the precision may play an important role in stability analyses, as in the case of the system with the hardening spring ${ }^{(26)}$.

Therefore, the standard pattern of the characteristic of the softening Duffing system with a damper may be concluded to be Fig. 1, which has taken both symmetric and unsymmetric solutions simultaneously.

Figure 5 shows the influence of the external exciting force on the behavior of the system, where Fig. $5(\mathrm{a})$ is approximate solutions and Fig. $5(\mathrm{~b})$ is numerical solutions. Note that the stability analysis of Fig. 5( a ) has been based upon expansion method with 5 th order. From these figures, it is clear that, as

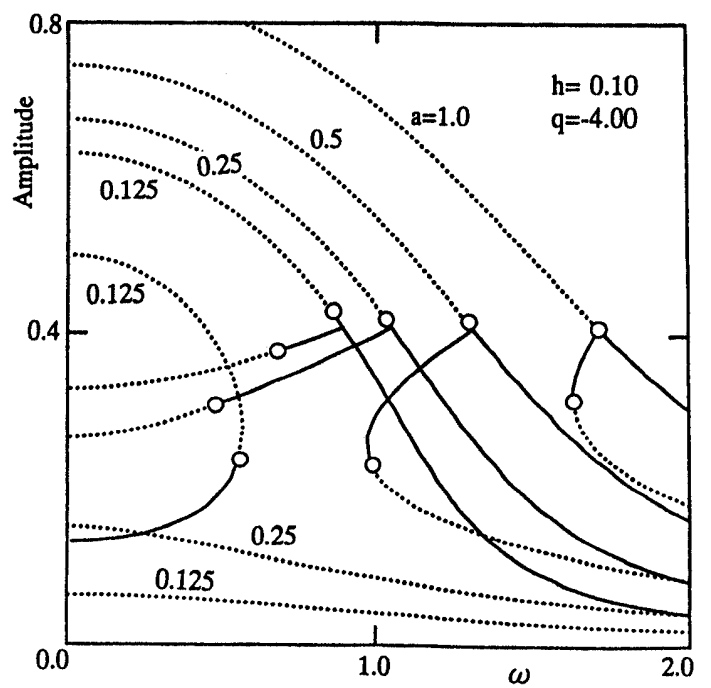

(a ) "Approximate solutions"

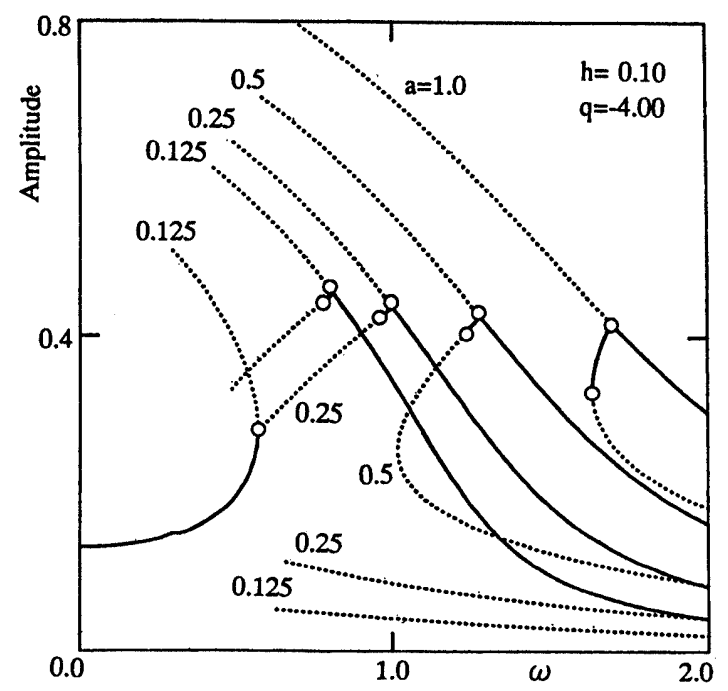

(b) "Numerical solutions"

Fig. 5 Character of resonance curves at various values of the external force 
the external force increases, the unsymmetric branch (2) will be connected with the branch (3) and also the width of the stable region on the branch (2) will expand gradually. In addition, the stability of approximate solutions may agree qualitatively and quantitatively quite well with one of numerical solutions.

From Figs. 1 to 5, the results of previous works ${ }^{(4),(5),(10),(12)}$ seem to be insufficient in order to express precise behaviors of the system. That is, as to the fundamental harmonic solution, Eq. (2) is both uncertain and unsatisfactory, and therefore Eq. (3) has to be adopted. It is worth noticing that the branches (2) and (3) of unsymmetrical solutions can be obtained even by the rough approximate solution such as Eq. (3), and the stable region on the branch (2) can be easily predicted. Furthermore, it is also clear that only expansion method can provide precise and reliable analyses of stabilities of Eqs. (2) and (3).

Therefore, the procedure suggested may result in the improvement of previous stability analyses about harmonic response curves, i.e., branches (1) and (2), except for the basic pattern of harmonic response curves.

\subsection{Interior structure of main resonant region and subharmonic solutions}

The specific behaviors of the system have been examined by using the numerical, analytical procedure ${ }^{(32)}$, with the exception of the responses of the harmonic motions discussed in the section 3.1. The response curves based upon the numerical scheme, i.e., the numerical solutions, are drawn in Fig. 6 , where the marks 2-T, 3-T correspond to the 2nd, 3rd subharmonic oscillations, respectively. The figures indicate that there may exist many kinds of subharmonic oscillations in the main resonant region. The details of the region, near the section $A B C$, enclosed with the branches (1) and (2) in Fig. 6 is shown in Fig. 7, where $q=-4.0$ in the case of Figs. 7( a ) and (b) , and $q=-3.0$ in Figs. 7 (c) and (d). By looking at Fig. $7(\mathrm{a})$, as the frequency $\omega$ decreases $(\mathrm{A} \rightarrow \mathrm{B})$ on the branch (2), we can see that the state of the branch (2) becomes unstable at point $\mathrm{B}$, and the state of the branch (2) remains unstable beyond the point $\mathrm{B}$ (i.e., in the region $\mathrm{BD}$ ).

On the other hand, the new branch with twice the period of the state on the region $\mathrm{AB}$, which corresponds to the state: $\mathrm{BE}$, appears. Thus, as the frequency $\omega$ decreases further, this unsymmetric branch $\mathrm{BE}$ also becomes unstable and another new branch with twice the period of the state of the region $\mathrm{BE}$ results in appearance (i.e., period doubling bifurcation). However, it is clear that there may exist many response curves with a peninsular shape in the triangular region $\mathrm{ABC}$ in addition to the branches $\mathrm{AB}$ and $\mathrm{AC}$ as shown in Fig. 7. They correspond to the motions with $3-\mathrm{T}, 4-\mathrm{T}$ and possess a narrow state at the apex of each response curve. The variation of the coefficient of a nonlinear spring has qualitatively little influence on the state of their existence, while the alteration of the damping coefficient has a little influence on the frequency region of their existence.

Varying the system parameters, the pattern of the response curves will change from the form as shown in Figs. 1 and 5 into the one of Fig. 8, where the branch (1) of Figs. 1 and 5 divides into the upper and lower branch (see Fig. 2 in Refs. (4) and (5)). Figure $8(\mathrm{a})$ shows the qualitative response curves obtained through the approximate analysis, and Fig. 8(b) expresses the details of the upper branch of numerical solutions. The lower branch is omitted, because it is quite simple. The bifurcation phenomenon into the



(a) "Numerical solutions" at $h=0.1$

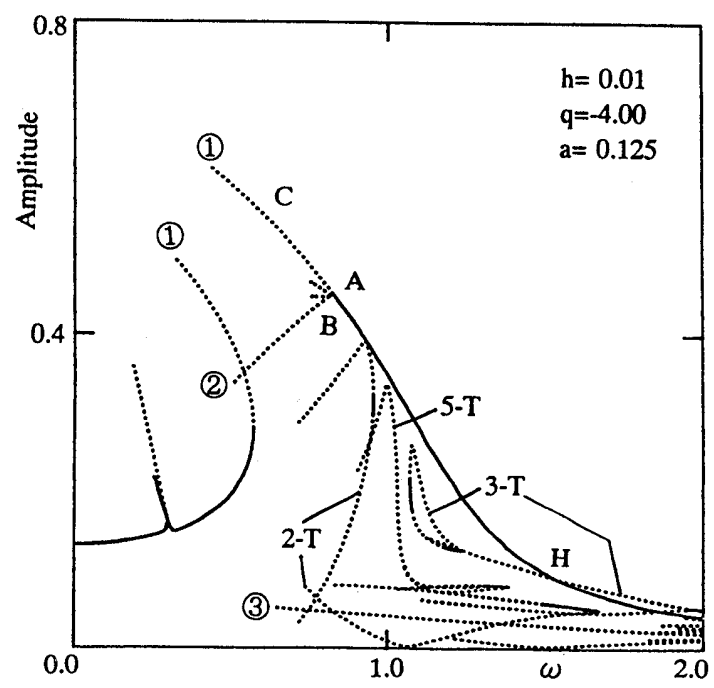

(b) "Numerical solutions" at $h=0.01$

Fig. 6 Details of response curves 


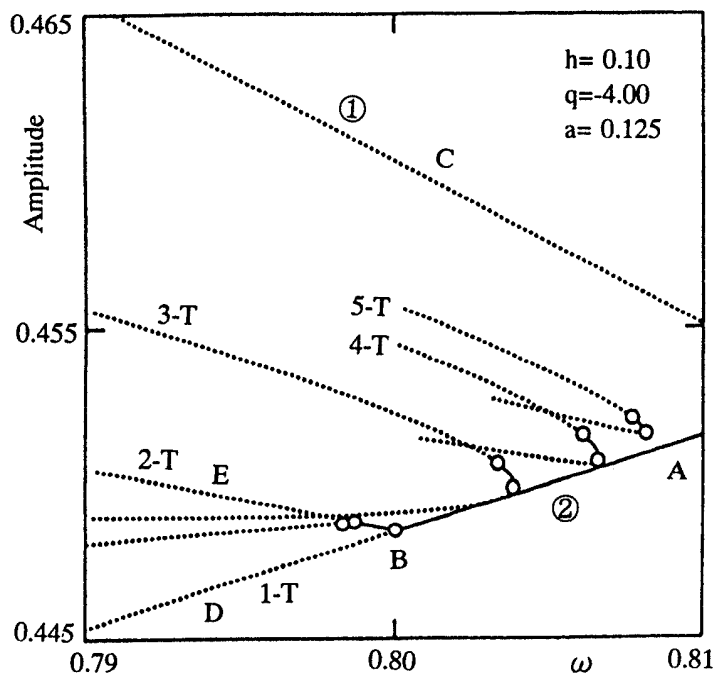

(a) The case of $q=-4.0$ and $h=0.1$

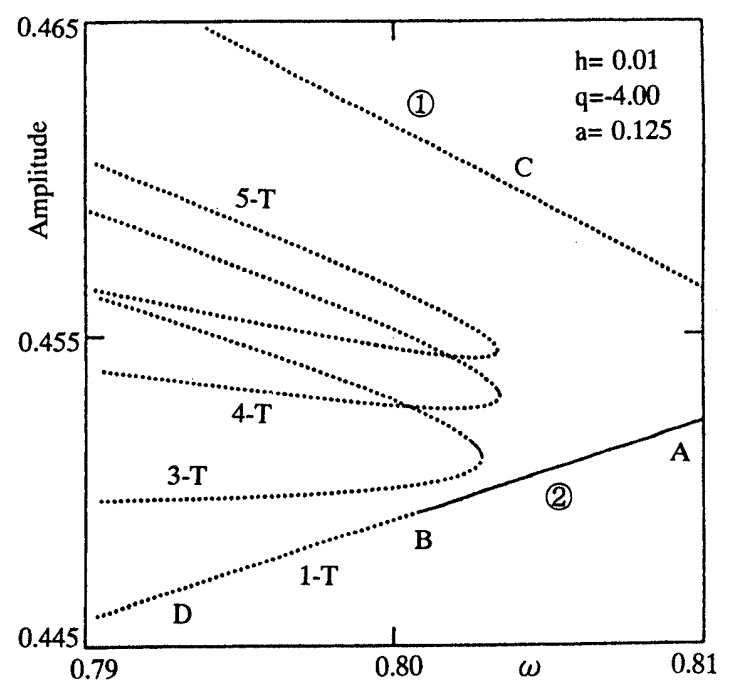

(b) The case of $q=-4.0$ and $h=0.01$

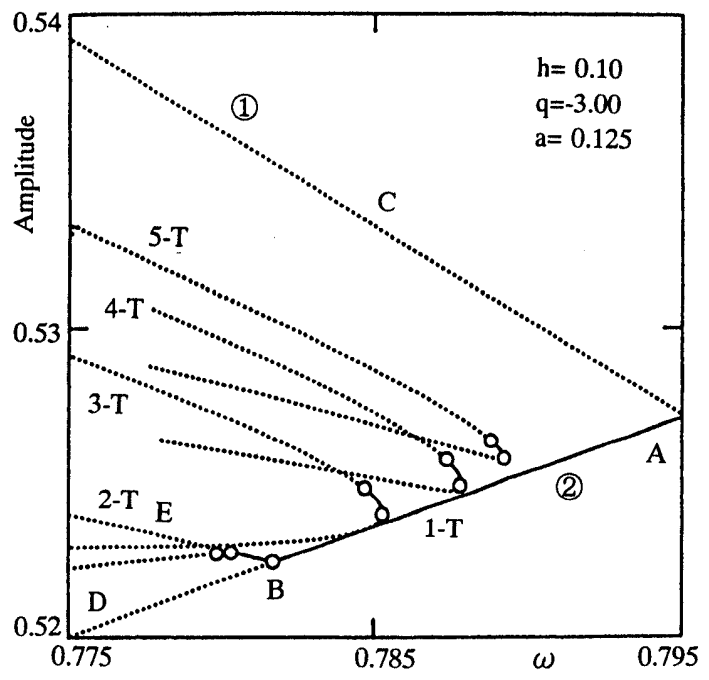

(c) The case of $q=-0.3$ and $h=0.1$

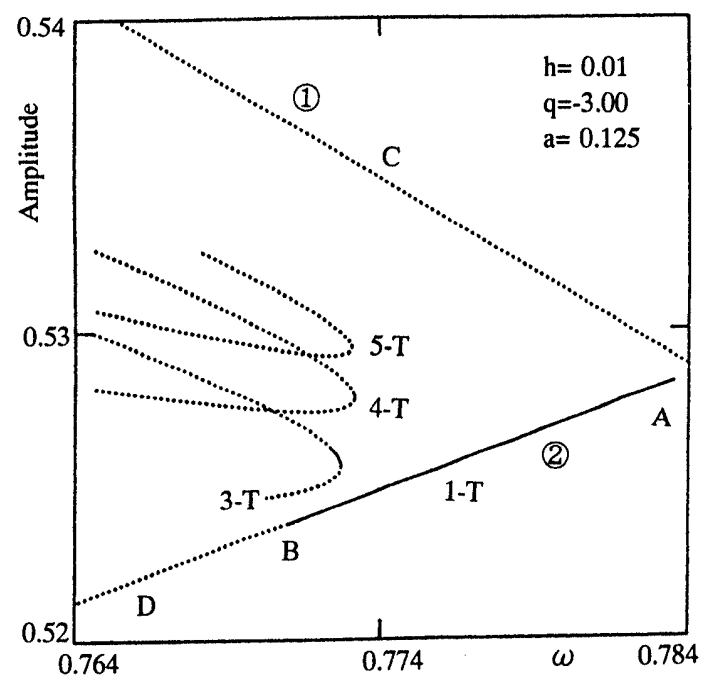

(d) The case of $q=-3.0$ and $h=0.01$

Fig. 7 Response curves (: numerical solutions) near the bifurcating point in resonant region

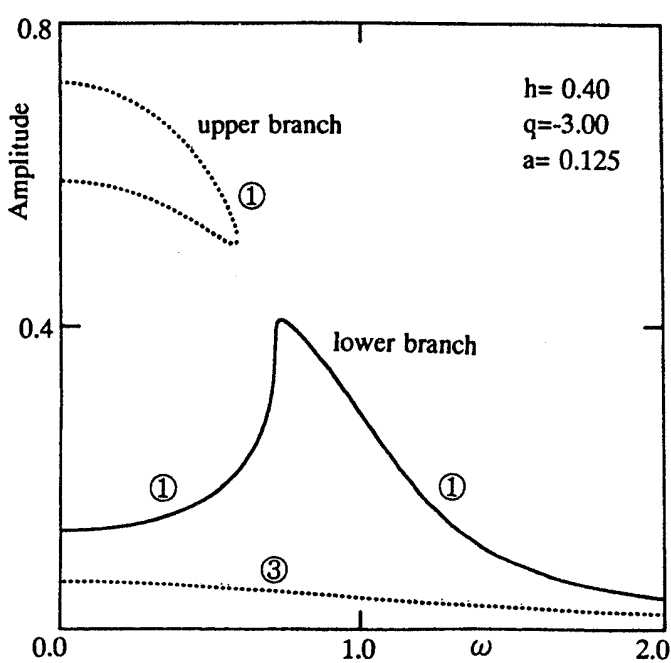

(a ) "Approximate solution"

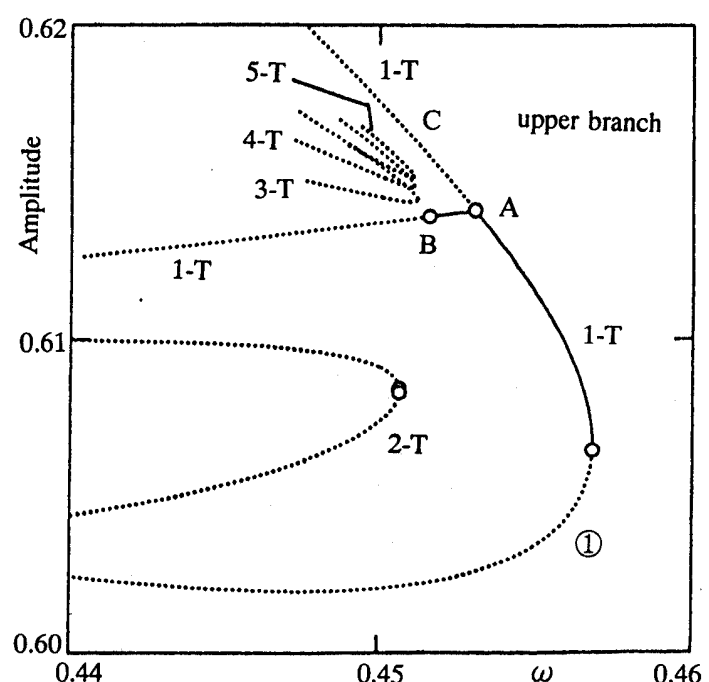

(b) Details of the upper branch suing the numerical procedure

Fig. 8 Resonance curves of the harmonic solution 
branches (1) and (2) at the point A, and the existence of the stable region on the branch (2) may be analogous to the characteristic of Figs. 1 and 5.

\section{3 Unsymmetric solutions and chaotic phenom- ena}

In the following section of this paper, the structure of these solutions has been analyzed on the phase plane. In Fig. 9, the integral curves, i.e., fixed points (in Fig. 9(a)) and the basin of attraction (in Fig. $9(\mathrm{~b})$ ) for the fundamental harmonic solutions are shown on the phase plane, where the black region corresponds to the initial one leading to this solution and the blank region to the initial one leading to unbounded solutions at the frequency: $\omega=1.0$.

Referring to Fig. 1, the resonant harmonic solution may only exist at the frequency, and this solution corresponds to the stable spiral fixed point as shown with the symbol: $P_{0}$ in Fig. 9(a), where solid lines

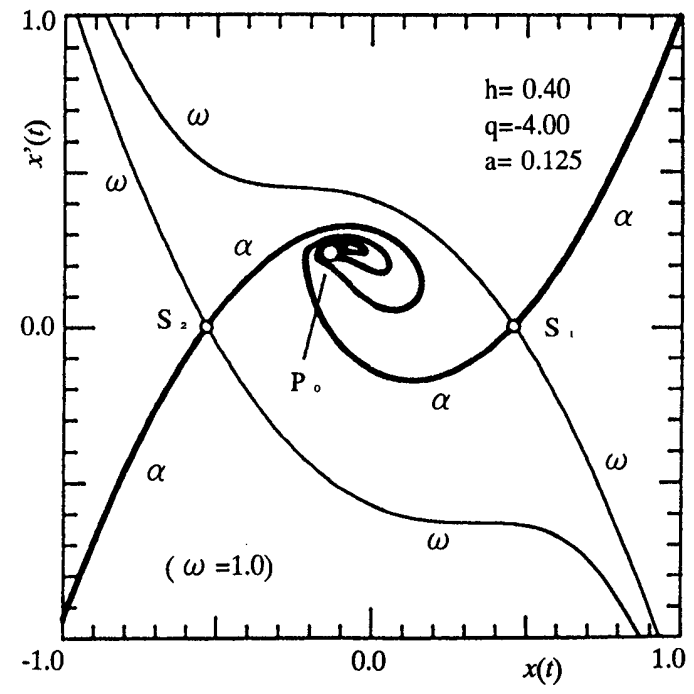

(a) Invariant curves in the phase plane

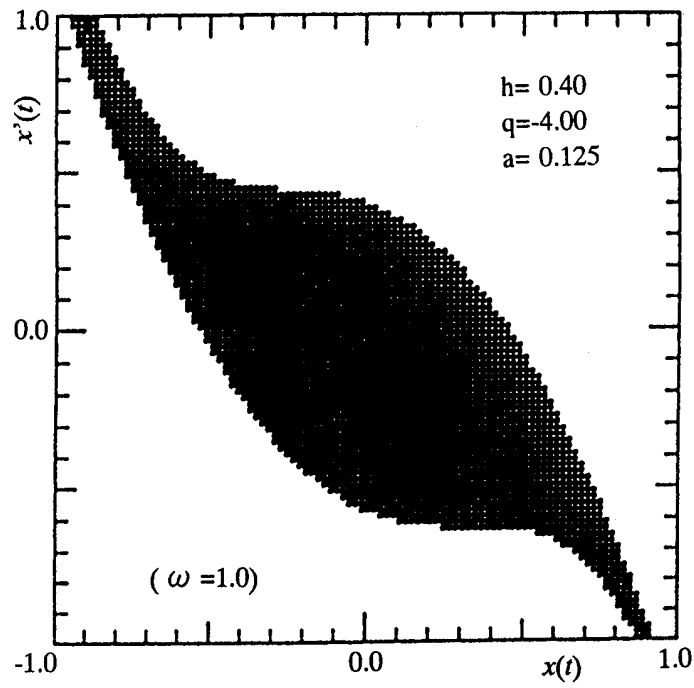

(b) Basin of attraction

Fig. 9 Characteristic curves of the harmonic solution and thin lines mean unstable invariant curves (i.e., $\alpha^{-}$ branch) and stable ones (i.e., $\omega$-branch), respectively. On the other hand the response curves presented in previous papers ${ }^{(3)-(7),(10)}$ such as Fig. 1 without the branch (3) could not explain reasonably the fact that the attractor leading to this solution becomes a shape similar to that of "a human stomach". Unless there do exist saddle points with the marks: $S_{1}$ and $S_{2}$ in Fig. 9 , one can not construct a resonable attractor leading to this solution. As a matter of fact, these two unstable saddle points, $S_{1}$ and $S_{2}$ correspond to the unstable branch (3) in Fig. 1 and this branch (3) is unsymmetrical as well. Therefore, this branch (3) has two different points, which mean both a positive and negative solutions, as shown in the phase plane.

Schmidt \& Tondl $^{(7)}$ have possibly presented the contents similar to the results of Fig. 9, as far as the authors know. Although only the results for the attractor (i.e., the shape like a stomach) may be considered valid, they did not clearly explain separatrices, which separate the stable regions from the unstable ones, which are accompanied with saddle points. It is impossible to clarify rationally the basins of attraction in the main resonant region until the existences of such solutions $\left(S_{1}\right.$ and $S_{2}$, joined with the unstable unsymmetric branch (3)) have been definitely verified.

The initial regions leading to the stable solutions on the unsymmetric branch (2) in Fig. 1 are shown in Fig. 10. The symbol " $\mathbf{\Delta}$ " means the initial region leading to the positive unsymmetric solution, corresponded to the solution with $R>0$ in Eq. (3), while the mark " $\boldsymbol{\nabla}$ " means the region leading to the negative one with $R<0$. As the frequency further

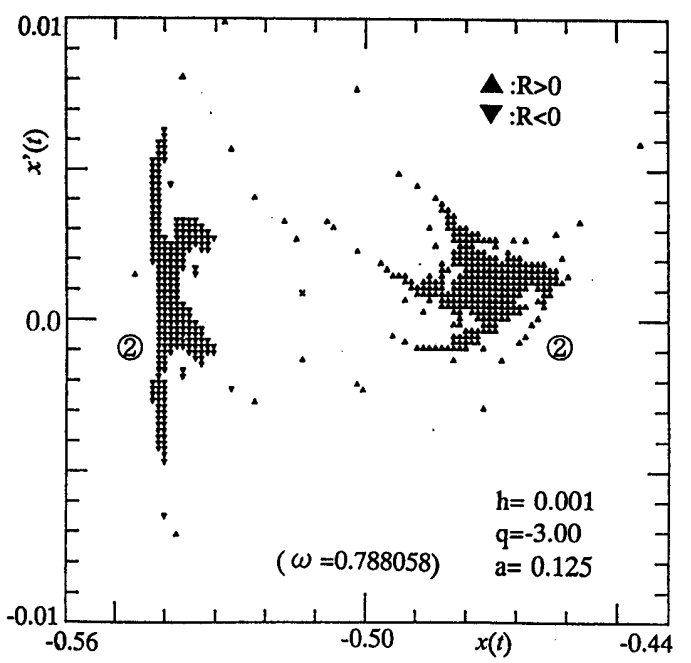

Fig. 10 Initial regions leading to the unsymmetrical harmonic solution

A : positive unsymmetrical solution,

$\boldsymbol{\nabla}$ : negative unsymmetrical solution 
decreases from the state as shown in Fig. 10, the response will become more complex and result in chaotic via period doubling bifurcations, clarified by previous papers, just along the branch (2).

As shown in Figs. 1, 3 and 7, chaotic phenomena, which are accompanied with the fundamental harmonic solution in the frequency region of interest, can not occur along the path of the symmetric branch (1) (i.e., section $\mathrm{CA}$ ), but the unsymmetric branch (2) (i.e., section $\mathrm{AB}$ ). References (11),(12) and (33) may be questionable and erroneous with regard to this process. When the frequency decreases further from the state with Fig. 10 (i.e., section B $\rightarrow$ E in Fig. 7), the behavior is shown in Fig. 11, where Fig. 11 (a) is the bifurcation diagram and Fig. 11(b) is the invariant curves under the chaotic. The broken line in Fig. 11 (a) indicates the unstable state $\mathrm{AC}$ on the symmetric branch (1), i.e., the locus of saddle points, and then this branch $\mathrm{AC}$ has only something to do with crises of $\operatorname{chaos}^{(34)}$.

Figure 12 shows the details of the response curve with period 2-T (i.e., unsymmetric solution) as shown

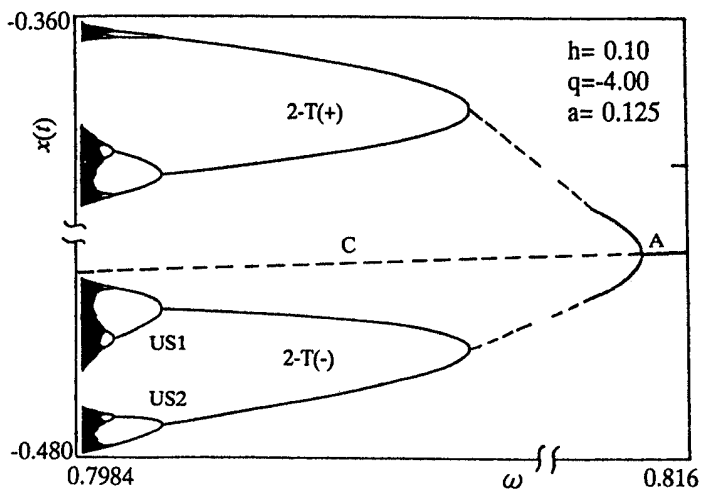

(a) Bifurcation diagram

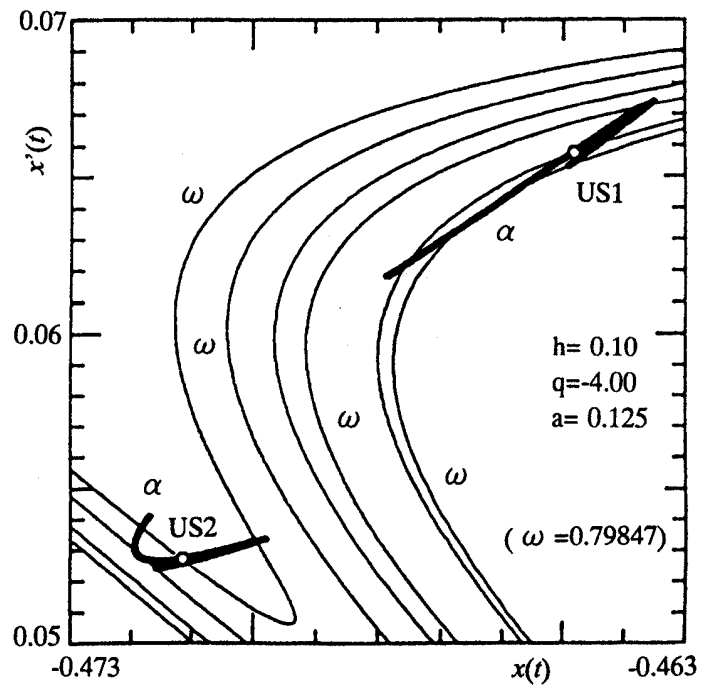

(b) Invariant curves

Fig. 11 Characteristics under chaotic motion in Figs. 7 ( c) and (d). The branch (1) corresponds to that of the symmetric, resonant solution in Figs. 7( c) and $(\mathrm{d})$. It shows that the solution with period 2-T contains quite a narrow stable region and therefore it can coexist with the harmonic solution. Figure 13 is the basin of attraction ${ }^{(35)}$ for the resonant region where the solution with period 2- $\mathrm{T}$ coexists with the harmonic solution as seen in Fig 12. Figures 13(b) and (d) correspond to the details of Figs. 13 (a ) and (c), respectively. In the figure, the mark "." signifies the initial region leading to the harmonic solution, the symbol "+" means one leading to a positive unsymmetric solution and the mark " $x$ " signifies one leading to a negative unsymmetric solution. Compared with the initial region attracted to the harmonic solution, the region leading to the solution with period 2-T is very sparse, and also the basin of boundaries of both initial regions have developed entanglements and the boundaries have possibly become fractal, as seen in Figs. 13(b) and (d).

Figure 14 shows the characteristics under the state where the solution with period $5-\mathrm{T}$ is unstable, and Figs. 14 ( a ) and ( b ) show the invariant curves, where thick lines correspond to unstable manifolds and thin lines to stable ones, and the initial region leading to the harmonic solution, respectively. The basin boundary of Fig. 14 ( b ) develops fingers, as in the same case of Fig. 13(c). While Ref.(33) also points out that the basin boundary develops fingers as the value of the forcing parameter varies, the substances of these fingers are not so simple. That is, the formation of fingers of Fig. 13 is affected by the existence of the solution with period $2-T$, whereas one of Fig. 14 is influenced by the solution with period $5^{-}$ $\mathrm{T}$. Therefore, it is noted that basin-boundary metamorphoses in the region of interest is not homogenous.

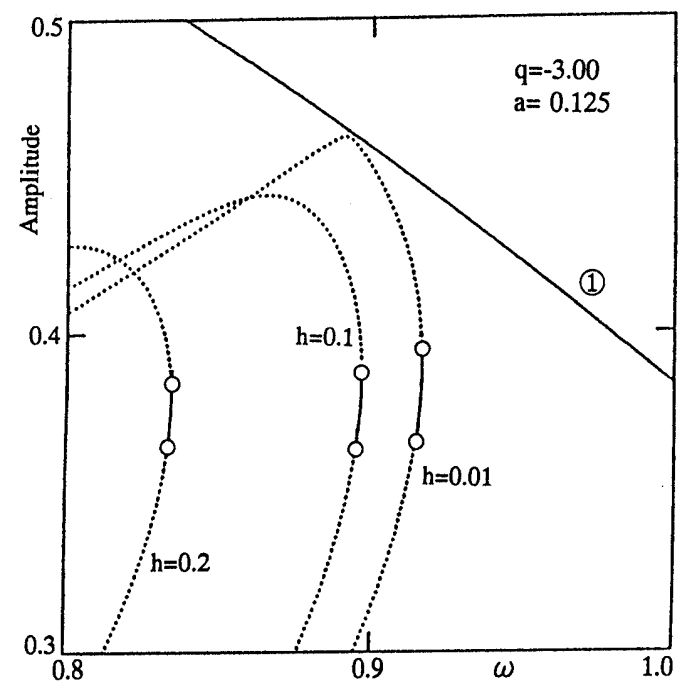

Fig. 12 Subharmonic solution of order $1 / 2$ (i.e., $2-\mathrm{T}$ ) 


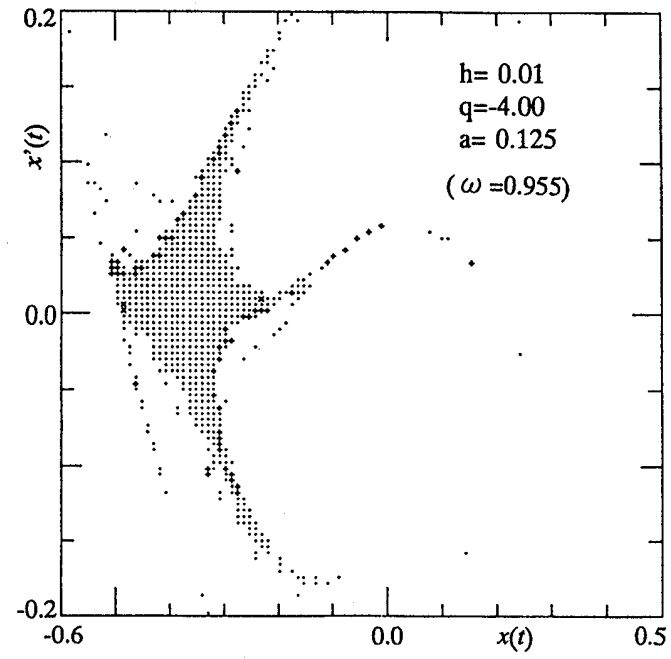

(a) Basin of attraction: $\omega=0.955$

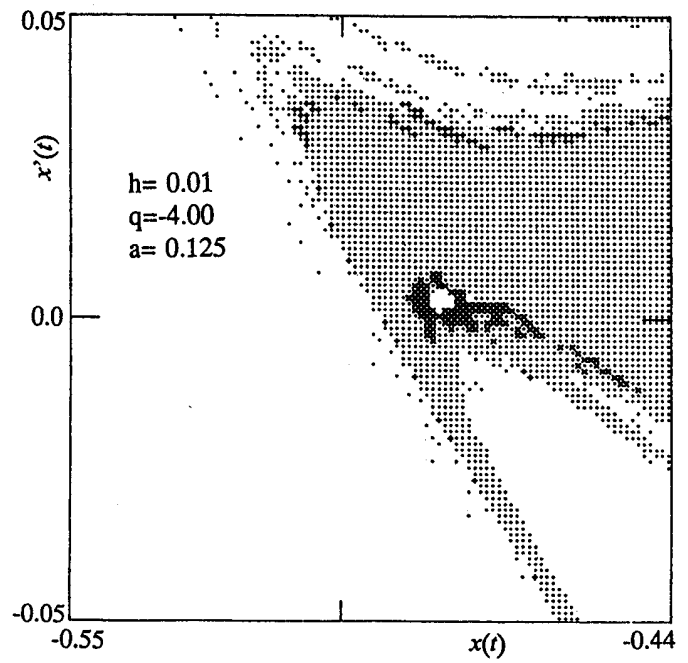

(b) Details of Fig. 13(a)

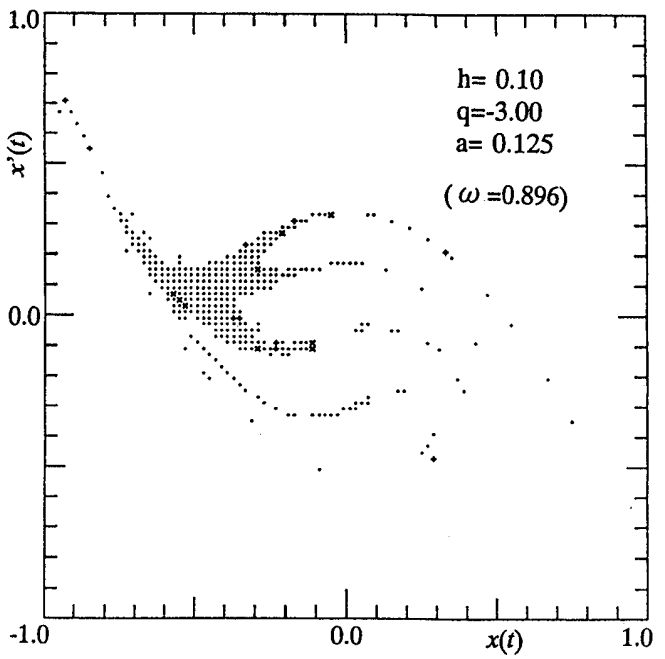

(c) Basin of attraction: $\omega=0.896$

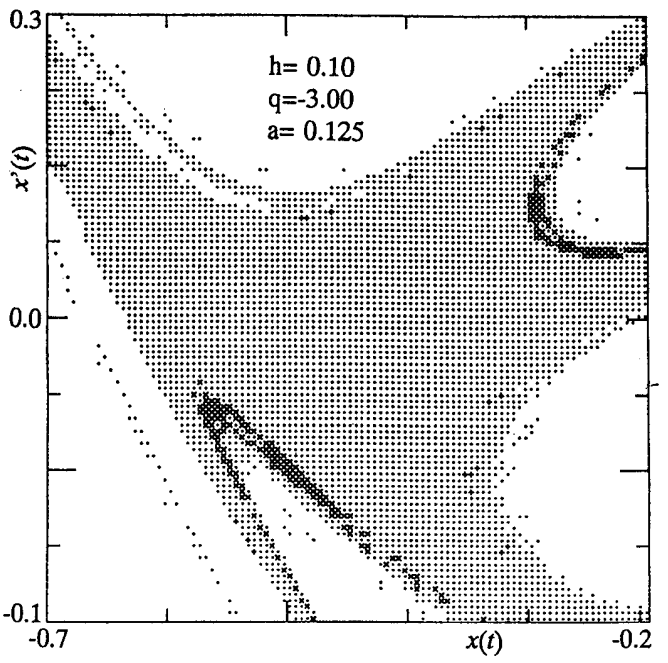

(d) Details of Fig. 13(c)

Fig. 13 Basins-boundary metamorphoses for the resonant region

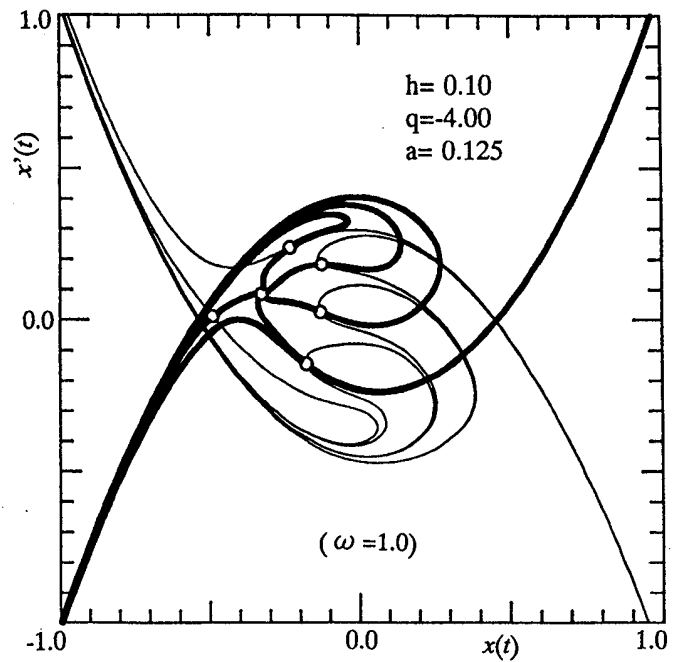

(a) Invariant curves

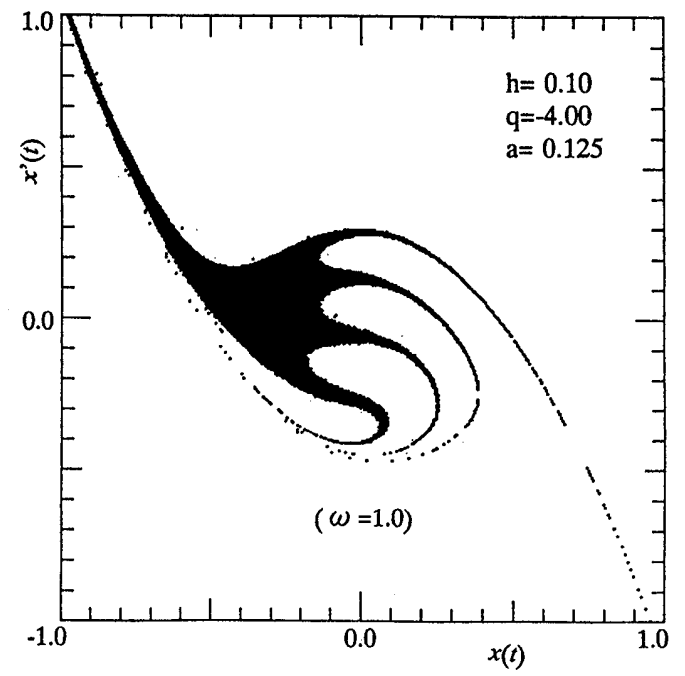

(b) Basin of attraction for the harmonic solution

Fig. 14 Basin structures 


\section{Conclusions}

Nonlinear oscillators of the damped Duffing's systems with a softening spring, which are fundamental and important for nonlinear vibrating phenomena, have been numerically investigated and the behaviors and stability analyses in the region of the principal resonance have been analyzed by means of the first approximate solution and the numerical integration method.

It has been shown that, in addition to the known solutions, i.e., symmetric responses, there exist unsymmetric solutions and many kinds of subharmonic solutions. Also a precise stability analysis was developed. The conclusions are as follows:

(1) There exist unsymmetric solutions, in addition to symmetric resonant and nonresonant solutions previously presented.

(2) The characteristics and basin boundaries of attraction can not be reasonably explained in the main resonant region until both symmetric and unsymmetric solutions have simultaneously been examined.

(3) Approximate stability analyses can analyse the stabilities of both these solutions. The analyses may supply improved results, compared with the results of stabilities of previous papers.

(4) The relation between the order of approximation of approximate solutions and that of approximate stability analyses has been clarified. The approximate stability analyses of the softening system need a higher order of approximation compared to the hardening system.

( 5 ) When the frequency decreases, the fundamental harmonic solution (which is symmetric) exhibits symmetric breaking phenomenon at some frequency. As a result, an unsymmetrical solution appears from this symmetric one. Phenomena of period doubling bifurcation and chaos, clarified by previous papers, can only occur along the path of the unsymmetrical one, but not the path of the symmetric one.

(6) In the frequency region, that is, both the sides lying near the branch of the unsymmetric solution, many kinds of subharmonic solutions occur, which are isolated solutions. Although the width of the stable state is very narrow, each of these subharmonic solutions possesses a stable state.

\section{References}

(1) Dowell, E.H. and Pezeshki, C., J. of Sound \& Vibration, Vol. 121, No. 2 (1988), p. 195.

(2) Novak, S. and Frehlich, R.G., Phys. Review A, Vol. 26, No. 6 (1982), p. 3660.

(3) Stoker, J.J., Nonlinear Vibrations in Mechanical and Electrical Systems, (1950), p. 83, Interscience
Publ.

(4) Leung, A.Y.T., J. of Sound \& Vibration, Vol. 149, No. 1 (1991), p. 147.

(5) van Dooren, R., J. of Sound of Vibration., Vol. 123, No. 2 (1988), p. 327.

(6) Hubermann, B.A. and Crutchfield, J.P., Phys. Review Letters, Vol. 43, No. 23 (1979), p. 1743.

( 7 ) Schmidt, G. and Tondl, A., Non-Linear Vibrations, (1986), p. 245, Cambridge Univ. Press.

(8) Tamura, H., Konndou, T., Sueoka, A. and Ueda, N., Trans. Jpn. Soc. Mech. Eng., (in Japanese), Vol. 52, No. 473, C (1986), p. 40.

(9) Pezeshki, C. and Dowell, E.H., J. of Sound \& Vibration, Vol.117, No.2 (1987), p. 219.

(10) Szemplinska-Stupnicka, W., Int. J. of Non-Linear Mech., Vol. 23, No. 4 (1988), p. 257.

(11) Dowell, E.H. , Murphy, K.D. and Katz, A.L., Nonlinear Dynamics, Vol. 6 (1994), p. 247.

(12) Szemplinska-Stupnicka, W., Nonlinear Dynamics, Vol. 3 (1992), p. 225.

(13) Szemplinska-Stupnicka, W., Nonlinear Dynamics, Vol. 7 (1995), p. 129.

(14) Thompson, J.M.T. and McRobie, F.A., Proceedings of the Int. Conference: 1st European Nonlinear Oscillatins Conference (ed., Kreuzer, E. and Schmidt, G.) (1993), p. 107.

(15) Thompson, J.M.T. and Bishop, S.R., Nonlinearity and Chaos in Engineering Dynamics, (1994), p. 3, Wiley.

(16) Taniguti, O. (ed.), Vibration Engineering Handbook (in Japanese), Youkendou (1976), p. 411.

(17) Yamamoto, T., Yasuda, H. and Nagasaka, I., Trans. Jpn. Soc. Mech. Eng., (in Japanese), Vol. 42, No. 356, No. 1 (1976), p. 1071.

(18) Guckenheimer, J. and Holmes, P., Nonlinear Oscillations, Dynamical Systems, and Bifurcations of Vector Fields, (1983), p. 174, SpringerVerlag.

(19) Hayashi, C., Nonlinear Oscillations in Physical Systems, (1964), p. 82, McGraw-Hill.

(20) Lakshmanan, M. and Murali, K., Chaos in Nonlinear Oscillators, (1996), p. 67, World Scientific.

(21) Furuya, S. and Nagumo, J., Theory of Nonlinear Vibration (in Japanese), (1957), p. 49, Iwanamishoten.

(22) Saitou, T., Ordinary Differential Equation I (in Japanese), (1976), p. 17, Iwanamishoten.

(23) Mathematical Society of Japan, Mathematical Dictionary (in Japanese), (1974), p. 491, Iwanamishoten.

(24) Nayfeh, A.H. \&Bakachandran, B., Applied Nonlinear Dynamics, (1995), p. 158, John Wiley\&Sons.

(25) Argyris, J., Faust, G. and Haase, M., An Exploration of Chaos, (1994), p. 159, North-Holland.

(26) Hamdan, M.N. and Burton, T.D., J. of Sound \& Vibration, Vol. 166, No. 2 (1993), p. 255.

(27) Bolotin, B.B., Dynamical Stability in Elastic System (in Japanese), (1982), p. 1, Korona-sha.

(28) Maezawa, S., Nonlinear Ordinary Differential Equation (in Japanese), (1969), p. 30, Daiyamondo- 
sha.

(29) Hassan, A., Nonlinear Dynamics, Vol. 10 (1996), p. 105.

(30) Hassan, A. and Burton, T.D., J. of Sound and Vibration, Vol. 182, No. 4 (1995), p. 523.

(31) Satou, H. , Trans. Jpn. Soc. Mech. Eng., (in Japanese), Vol. 39, No. 318, NO. 1 (1973), p. 610.

(32) Tsuda, Y., Tamura, H., Sueoka, A. and Fujii, T., Chaotic Behavior of a Nonlinear Vibrating Sys- tem with a Retarded Argument, JSME Int. J., Ser. III, Vol. 35, No. 2 (1992), p. 259.

(33) Nayfeh, A.H. and Sanchez, N.E., Int. J. of NonLinear Mech., Vol. 24, No. 6 (1989), p. 483.

(34) Grebogi, C., Ott, E. and Yorke, J.A., Phys. Review Letters, Vol. 48, No. 22 (1982), p. 1507.

(35) Hsu, C.S. and Guttalu, R.S., ASME, J. of Appl. Mech., Vol. 47 (1980), p. 940. 